\title{
Percolating metallic structures templated on laser - deposited carbon nanofoams derived from graphene oxide: Applications in humidity sensing
}

\author{
Sebastian Nufer, ${ }_{1}^{1,2}$, Jonathan P. Salvage ${ }^{3}$, Aleksey Shmeliov ${ }^{4}$, Adam Brunton ${ }^{1}$, Alan B. Dalton ${ }^{2}$ \\ ${ }^{1}$ M-Solv Ltd, Oxonian Park, Langford Locks, Kidlington, Oxford, OX5 1FP, UK \\ 2 University of Sussex, Brighton, BN1 9RH, UK \\ 3 University of Brighton, Brighton, BN2 4GJ, UK \\ ${ }^{4}$ School of Physics, Trinity College Dublin 2, Ireland \\ Sebastian.nufer@m-solv.com
}

\begin{abstract}
Carbon nanofoam (CNF) is a low-density, high-surface-area material formed by aggregation of amorphous carbon nanoparticles into porous nanostructures. We report the use of a pulsed infrared laser to prepare CNF from a graphene oxide (GO) target material. Electron microscopy shows that the films consist of dendritic strings which form web-like three-dimensional structures. The conductivity of these structures can be modified by using the CNF as a nanostructured scaffold for gold nanoparticles deposited by sputter coating, controllably increasing the conductivity by up to four orders of magnitude. Upon decreasing humidity, the pristine CNF exhibits an increase in resistance with a quick response and recovery time. By contrast, the gold-sputtered CNF showed a decrease in resistance, indicating modification of the doping mechanism due to water adsorption. The sensitivity to humidity is eliminated at the percolation threshold of the metal on the CNF.
\end{abstract}

Key words: carbon nanofoam, humidity sensor, scaffold, specificity, selectivity
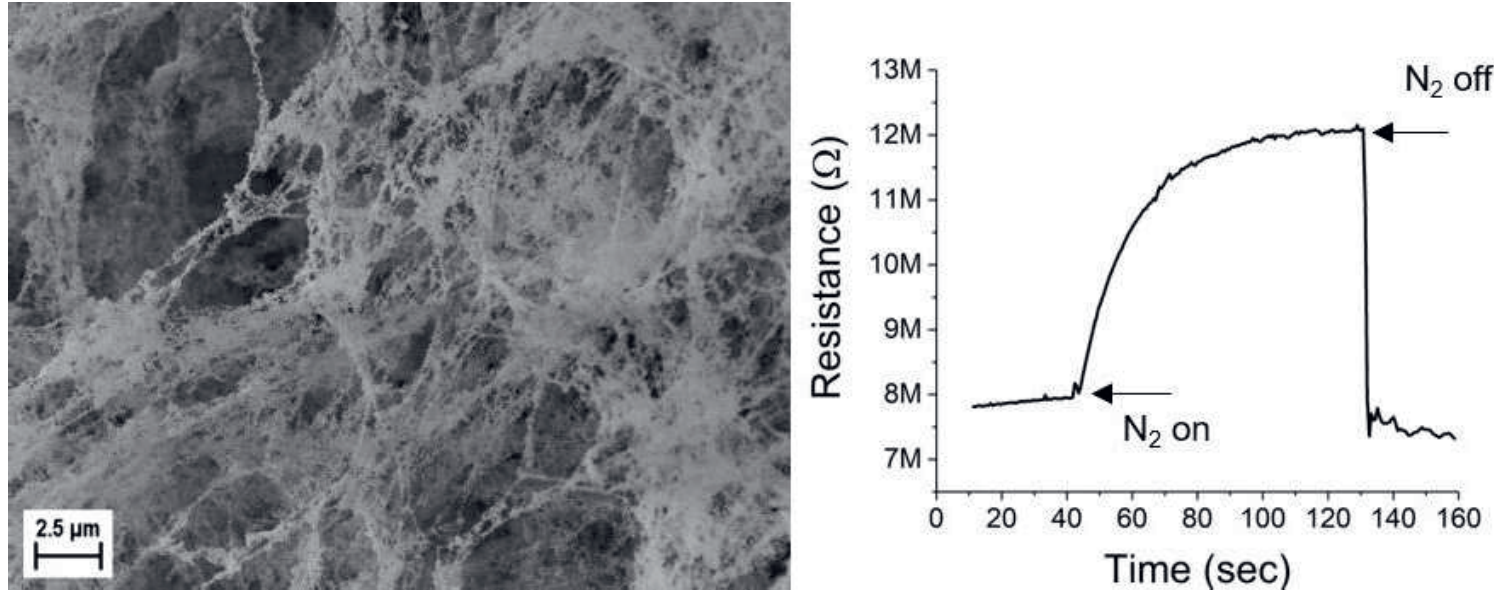

Figure 1: SEM of pristine CNF with the resistive signal when exposed to a dry air environment (changing from $37 \%$ rH to $20 \% \mathrm{rH}$ ) 

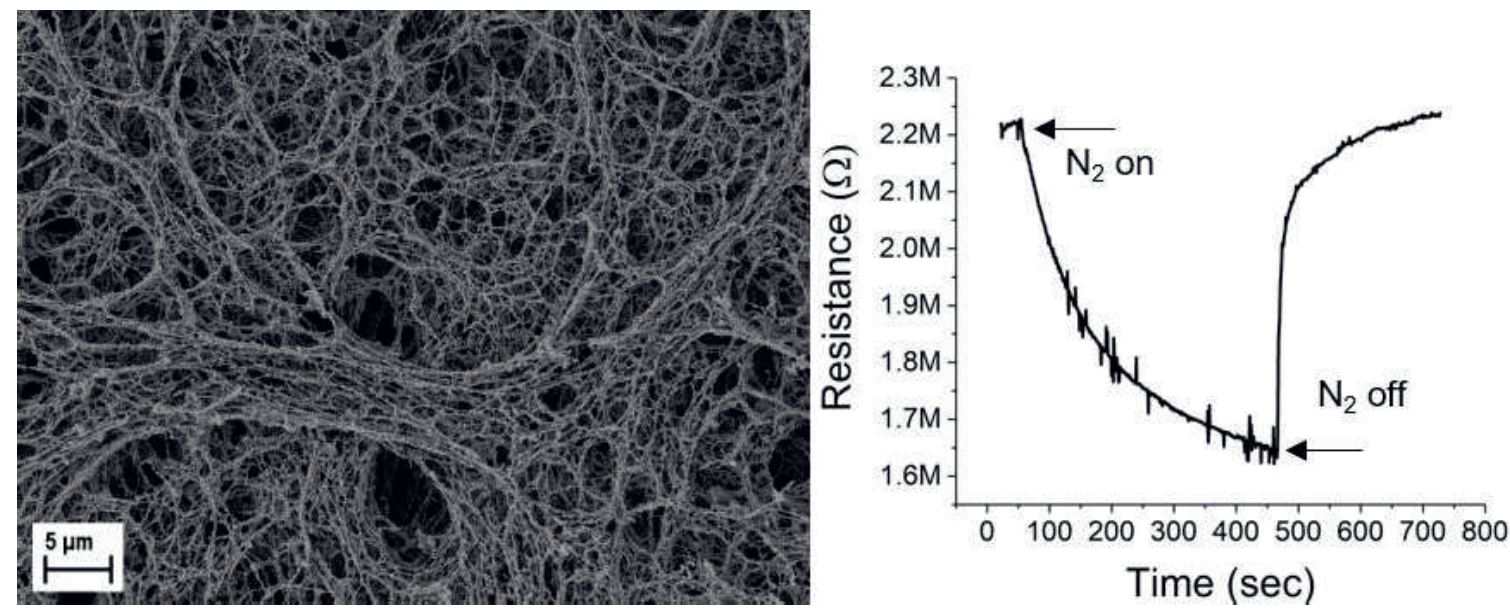

Figure 2: SEM of gold coated CNF with the resistive signal when exposed to a dry environment (changing from $37 \% \mathrm{rH}$ to $20 \% \mathrm{rH}$ ) resp. 